\title{
A New Class of Polymers: Starburst-Dendritic Macromolecules
}

\author{
D. A. Tomalia, ${ }^{*}$ H. Baker, J. Dewald, M. Hall, \\ G. KALlos, S. MARTIN, J. ROECK, \\ J. RYDER, and P. SMITH
}

\begin{abstract}
Functional Polymers/Process and *The Analytical Laboratory, Dow Chemical U.S.A., Midland, Michigan 48640, U.S.A.
\end{abstract}

(Received August 20, 1984)

\begin{abstract}
This paper describes the first synthesis of a new class of topological macromolecules which we refer to as "starburst polymers." The fundamental building blocks to this new polymer class are referred to as "dendrimers." These dendrimers differ from classical monomers/ oligomers by their extraordinary symmetry, high branching and maximized (telechelic) terminal functionality density. The dendrimers possess "reactive end groups" which allow (a) controlled moelcular weight building (monodispersity), (b) controlled branching (topology), and (c) versatility in design and modification of the terminal end groups. Dendrimer synthesis is accomplished by a variety of strategies involving "time sequenced propagation" techniques. The resulting dendrimers grow in a geometrically progressive fashion as shown: Chemically bridging these dendrimers leads to the new class of macromolecules - "starburst polymers" (e.g., $(\mathrm{A})_{n},(\mathrm{~B})_{n}$ or $\left.(\mathrm{C})_{n}\right)$.
\end{abstract}

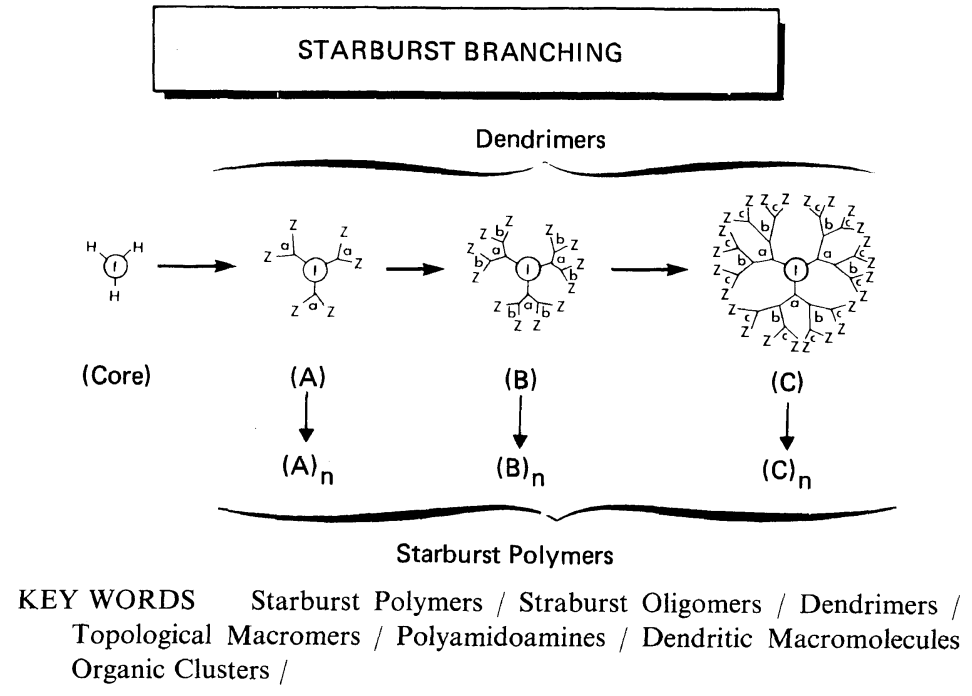

Considerable theoretical interest has been focused recently on molecular architecture possessing regular branching with radial symmetry. ${ }^{1-4}$ Although many important theoretical implications have evolved from examination of such models, actual synthesis of these prototypes has remained illustive.

We wish to report the first synthesis of such idealized models as shown in Figure 1, which we refer to as possessing "starburst" topology. ${ }^{5}$ These highly functionalized oligomers have been coined "dendrimers." Their successful synthesis and char- 


\section{A. Tomalia et al.}

acterization has allowed us to demonstrate the controlled occupation of space in three-dimensions as a function of size, shape and disposition of desired organic functionality.

Subsequent covalent bridging of these dendrimers produces a new class of topological polymers, with size controlled domains, which are referred to as "starburst" polymers.

We report here implications of the starburst concept, preliminary synthesis data and characterization results validating these unique structures.

\section{STARBURST BRANCHING CONCEPT}

Dendrimers differ from classical random coil macromolecules in that they possess three distinguishing architectural components, namely (a) an initiator core, (b) interior layers (generations), composed of repeating units, radially attached to the initiator core and (c) exterior (terminal functionality) attached to the outermost interior generation. Construction of these interior layers involves proceeding from initiator core $\rightarrow$ starbranched oligomer (generation $=1.0$ ) $\rightarrow$ starburst oligomers, as shown in Figure 1.

Ethylenediamine (EDA) and ammonia initiator cores were most extensively examined in this study. Their multiplicities, $\left(N_{\mathrm{c}}=4\right.$ or 3 , respectively), namely the number of active hydrogens, determines the number of branches in the starbranched oligomer intermediate which in turn are connected to the other interior generations. The interior layers (generations) consist of repeating units which are formally derived from $\mathrm{N}$-(2-aminoethyl) acrylamide, $\left(\mathrm{CH}_{2}=\mathrm{CH}-\stackrel{\mathrm{I}}{\mathrm{C}} \mathrm{NHCH}_{2} \mathrm{CH}_{2} \mathrm{NH}_{2}\right)$. These repeating units are constructed in a sequential fashion by (a) exhaustive Michael addition of an acrylate ester to an amine functionality to give subunit-1 and (b) amidation of the resulting ester moieties with an alkylene diamine (e.g., ethylene diamine) to give subunit-2. Completion of this sequence gives a full generation $(G=1.0)$ as shown below:

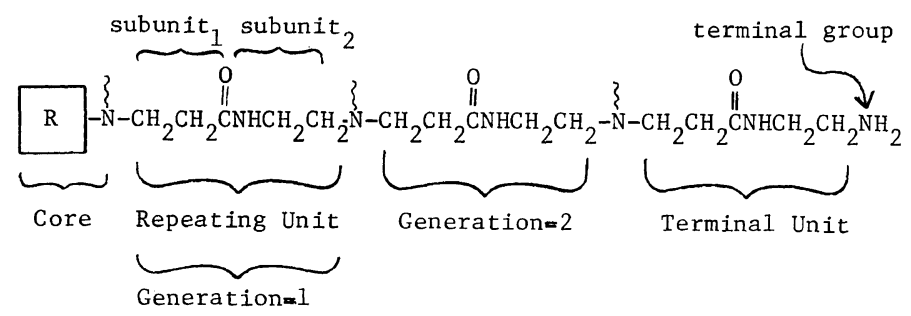

Interruption of this sequence at subunit- 1 leads to a half generation $(G=0.5)$. Repeating the above sequence leads to self-replication with concurrent introduction of repeating unit multiplicity $\left(N_{\mathrm{r}}=2\right)$ to yield generation $=2.0$. Termination at a half generation gives ester terminal groups, whereas full generations are terminated by amine functions.

Mathematically these multiplicities (i.e., $N_{\mathrm{c}}$ and $N_{\mathrm{r}}$ ) manifest themselves in the ordered development of branching and terminal functionality as a function of generation $(G)$, according to certain expressions below. A general structure notation for a monodispersed dendrimer with core functionality $N_{\mathrm{c}}$, repeating unit multiplicity $N_{\mathrm{r}}$, and generation $G$ is:

\section{[Core]}

$$
\left[\begin{array}{c}
\text { (Repeat unit) } \\
\frac{N_{\mathrm{r}}^{G}-1}{N_{\mathrm{r}}-1}
\end{array}{ }^{\text {(Terminal unit) }} N_{\mathrm{r}}^{G}\right]_{N_{\mathrm{c}}}
$$

Where: $N_{\mathrm{c}}=$ core multiplicity; $N_{\mathrm{r}}=$ repeating unit multiplicity.

In the notation of a particular dendrimer, retention of the subscripted terms in an unreduced form enables ready identification of all structural features from inspection of the structure notation. For example, a fifth generation dendrimer with repeating unit multiplicity 2 and core functionality 4 is best represented as:

$$
\begin{aligned}
& {\left[\mathrm{NCH}_{2} \mathrm{CH}_{2} \mathrm{~N}\right]} \\
& \quad\left[\left(\mathrm{CH}_{2} \mathrm{CH}_{2} \mathrm{CONHCH}_{2} \mathrm{CH}_{2} \mathrm{~N}\right)_{\left(2^{5}-1\right)} \mathrm{H}_{25}\right]_{4}
\end{aligned}
$$

By inspection of the structure notation, one can readily identify $N_{\mathrm{c}}, N_{\mathrm{r}}, G$, core structure, repeating unit structure, terminal structure, and calculate:

Number terminal units $=N_{\mathrm{c}} N_{\mathrm{r}}^{G}$

Polymer J., Vol. 17, No. 1, 1985 


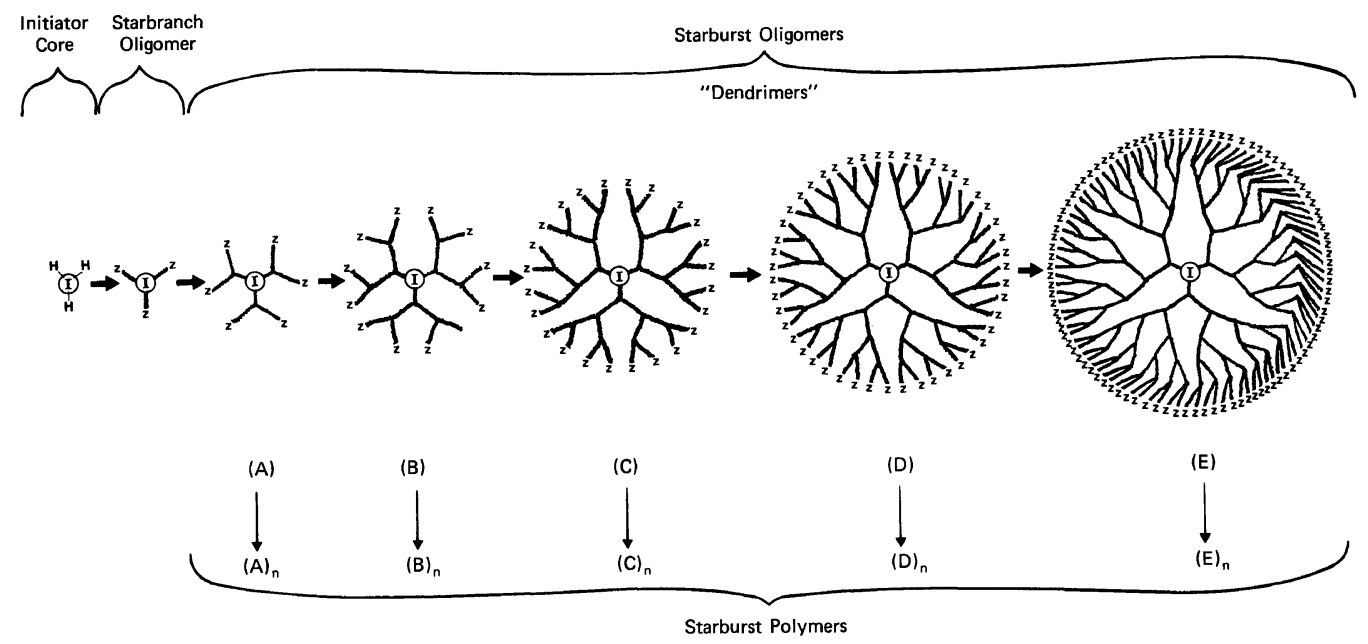

Figure 1. Starburst oligomers and their polymers.

Table I. Dendrimer features vs. generation

A $\left(\mathrm{NH}_{3}\right.$ core $)$

$$
\begin{aligned}
& \mathrm{N}\left[\begin{array}{cr}
\left(\mathrm{CH}_{2} \mathrm{CH}_{2} \mathrm{CONHCH}_{2} \mathrm{CH}_{2} \mathrm{~N}\right){ }_{N_{\mathrm{r}}^{G}-1}^{N_{\mathrm{r}}-1} & \frac{N_{\mathrm{r}}^{G}}{2}
\end{array}\right]_{3} \\
& \text { B (Ethylenediamine core) }
\end{aligned}
$$

\begin{tabular}{|c|c|c|c|c|}
\hline \multirow{2}{*}{ Generation } & \multicolumn{2}{|l|}{ A } & \multicolumn{2}{|l|}{ B } \\
\hline & $\begin{array}{l}\text { Molecular } \\
\text { weight }\end{array}$ & $\begin{array}{c}\text { Number of terminal } \\
\text { groups, } Z\end{array}$ & $\begin{array}{l}\text { Molecular } \\
\text { weight }\end{array}$ & $\begin{array}{c}\text { Number of terminal } \\
\text { groups, } Z\end{array}$ \\
\hline 0.5 & 275 & 3 & 404 & 4 \\
\hline 1.0 & 359 & 3 & 516 & 4 \\
\hline 1.5 & 875 & 6 & 1,204 & 8 \\
\hline 2.0 & 1,043 & 6 & 1,428 & 8 \\
\hline 2.5 & 2,075 & 12 & 2,804 & 16 \\
\hline 3.0 & 2,411 & 12 & 3,252 & 16 \\
\hline 3.5 & 4,475 & 24 & 6,004 & 32 \\
\hline 4.0 & 5,147 & 24 & 6,900 & 32 \\
\hline 4.5 & 9,275 & 48 & 12,404 & 64 \\
\hline 5.0 & 10,619 & 48 & 14,196 & 64 \\
\hline 5.5 & 18,875 & 96 & 25,204 & 128 \\
\hline 6.0 & 21,563 & 96 & 28,788 & 128 \\
\hline 6.5 & 38,075 & 192 & 50,804 & 256 \\
\hline 7.0 & 43,451 & 192 & 57,972 & 256 \\
\hline
\end{tabular}

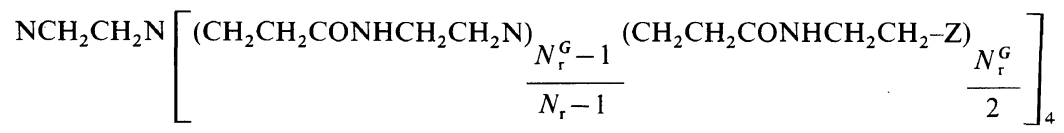




\section{A. Tomalia et al.}

Degree of polymerization $=N_{\mathrm{c}}\left(\frac{N_{\mathrm{r}}^{G}-1}{N_{\mathrm{r}}-1}\right)$

Molecular weight $=M_{\mathrm{c}}+N_{\mathrm{c}}\left(M_{\mathrm{r}}\left(\frac{N_{\mathrm{r}}^{G}-1}{N_{\mathrm{r}}-1}\right)+M_{\mathrm{t}} N_{\mathrm{r}}^{G}\right)$ where $M_{\mathrm{c}}, M_{\mathrm{r}}$, and $M_{\mathrm{t}}$ are the molecular weights of the core, repeating units, and terminal units, respectively. These equations allow one to readily calculate the number of terminal groups $(Z)$ or the theoretical molecular weight as shown in Table I.

\section{DENDRIMER SYNTHESIS}

Synthesis of the present polyamidoamine dendrimers is a two step process, involving; (a) exhaustive Michael addition of a suitable amine initiator core with methyl acrylate and (b) exhaustive amidation of the resulting esters with large excesses of ethylenediamine (EDA) as shown in Figure 2. This study focused primarily on ammonia and ethylenediamine as initiator cores. Dendrimer growth was examined through generation $=5.0$ $\left(\mathrm{NH}_{3}\right.$ core) and generation $=7.0$ (EDA core). Progress during both the alkylation and amidation steps was monitored by ${ }^{1} \mathrm{H}$ and ${ }^{13} \mathrm{C} \mathrm{NMR}$, size exclusion chromatography and infrared spectroscopy. Structure confirmation was obtained by chemical ionization and fast atom bombardment (FAB) mass spectroscopy as well as elemental analysis $(\mathrm{C}, \mathrm{H}, \mathrm{N})$ and will be discussed later. Under optimized conditions, material balances and yields were excellent (i.e., $98-100 \%$ ) and supported the stoichiometry expected for the dendritic structures.

More limited work showed that aromatic or aliphatic esters could also be used as initiator cores (e.g., $p$-methoxy benzoic acid methyl ester and methyl laurate). In these cases the above reaction sequence was reversed, giving an amine terminated dendrimer for (gen. $=0.5$ ) and an ester terminated dendrimer for $($ gen. $=1.0)$. Ethylenediamine was particularly suitable for the amidation step, since its

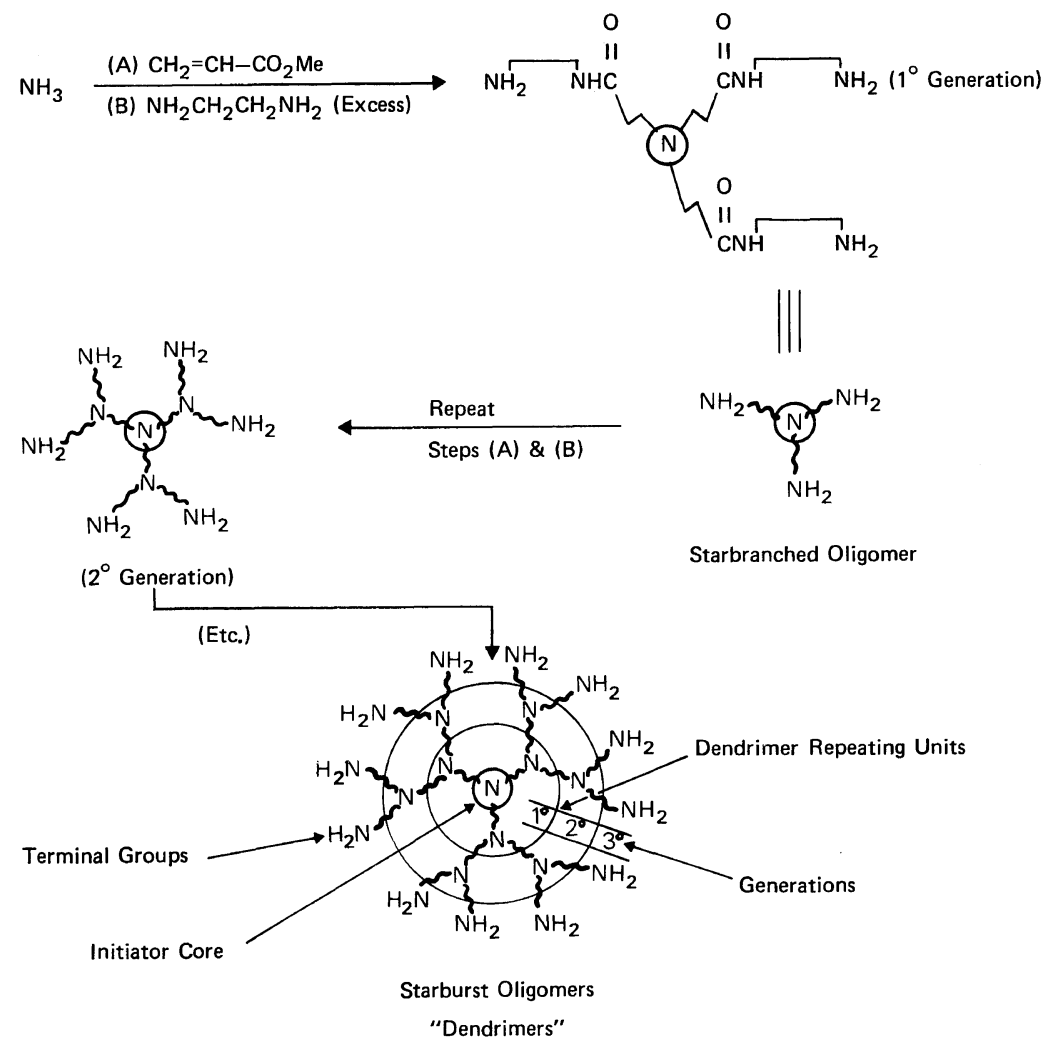

Figure 2. Dendrimer synthesis. 
boiling point (bp $110^{\circ} \mathrm{C}$ ) allowed removal of the large excesses under conditions which did not alter the dendrimer structure. Special azeotropic and wiped film distillation techniques were required to remove traces of EDA, especially in the higher generations.

In spite of these difficulties, a particle size analysis by electron microscopy of one of our dendrimers (core $=$ ammonia, generation $=4.5)$ showed it to be $\sim 87 \%$ monodispersed (i.e., $\pm 10 \%$ from $88 \AA$ ) and within $86 \%$ of the predicted dimension determined from CPK molecular models (i.e., $88 \AA$ actual vs. $76 \AA$ predicted). Synthesis of this dendrimer requires a nine step sequence which indicates (i.e., $X^{9}=0.87$ or $X=0.98$ ) that both the selectivity and yield of each step must be very high. Complete details concerning the scope and limitations of these dendrimer syntheses will be described elsewhere. ${ }^{7}$

\section{NON-IDEAL DENDRIMER GROWTH}

Digressions from non-ideal dendrimer growth are manifested in a variety of ways and include the following: (a) incomplete Michael addition reactions, (b) intramolecular cyclizations, (c) fragmentation due to retro-Michael reactions, and (d) solvolysis of terminal functionality. A methanolic medium was preferred for exhaustive Michael addition reactions. ${ }^{8}$ Attempts to conduct the addition reactions either neat or in non-protic medium invariable led to limited alkylation products. For example, neat alkylation of EDA gave predominately the tri- and tetra-adduct in a ratio of $\sim 70: 30$, whereas, in methanol tetra-adduct formation was

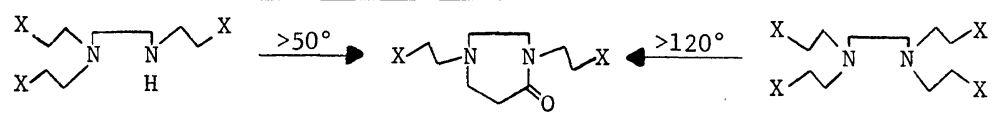

Where: $\quad \mathrm{X}=-\mathrm{CO}_{2} \mathrm{Me}$

essentially quantitative. The tri-adduct readily cyclizes to an azacaprolactam at temperatures $>50^{\circ} \mathrm{C}$ whereas the tetra-adduct requires temperatures $>100^{\circ} \mathrm{C}$. Cyclizations of this type were encountered whenever 1,2-alkylene polyamines were used as initiator cores and the chemistry will be described elsewhere. ${ }^{7}$ Another intramolecular cyclization reaction which produces macrocyclic defects has been observed sporadically under high dilution conditions by ${ }^{1} \mathrm{H}$ NMR spectroscopy. More characterization work is necessary to better define these unique defects.

Conducting both the alkylation and amidation steps at lower temperatures (i.e., $25-50^{\circ} \mathrm{C}$ ) are important. Higher temperatures (i.e., $>80^{\circ} \mathrm{C}$ ) are deleterious to ideal starburst branching not only due to intramolecular cyclization events but also because of retro-Michael reactions. It was found that first generation dendrimers ( core $=\mathrm{EDA}$ ) could be reversed at higher temperatures (i.e., $>100$ $120^{\circ} \mathrm{C}$ ) either in the presence or absence of the EDA amidating agent. A pivital intermediate, namely 2aminoethyl acrylamide was identified as a reactive fragment. In the absence of EDA it recombined with the fractured dendrimer to give branch extension, whereas in the presence of EDA it was trapped to give the amidotriamine as shown in Figure 3. Of course, subsequent dendrimer growth sequences lead to non-symmetrically branched (defective) dendrimers in either case. These events can dramatically affect the monodispersity of the dendrimers as one proceeds to higher generations.

Finally, it was observed that aqueous solutions of half-generation (ester terminated) dendrimers exhibited substantial surface active properties compared to full generation dendrimers. It was found that very facile hydrolysis to carboxylic acid groups accounted for this behavior. Precautions had to be taken to avoid exposure to moisture since the resulting carboxylic acid groups could not be forced to amidate under the usual conditions thus producing another type of branching defect.

\section{FACTORS AFFECTING DEGREE OF MONODISPERSITY}

Some dendrimer defect events (e.g., dendrimer fragmentation) can influence the degree of monodispersity during dendrimer growth. This is especially true if the fragments possess either or amine functions which may participate with the propagation sequencing reagents to produce new but "regressed dendrimer" entities. Other factors which also play a significant role include (a) dendrimer bridging and (b) incomplete removal of ethylenediamine at each 
D. A. Tomalia et al.

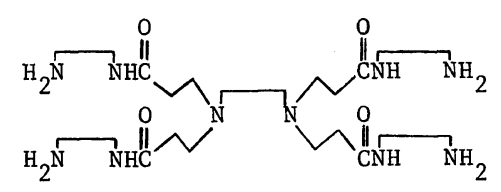

(A)

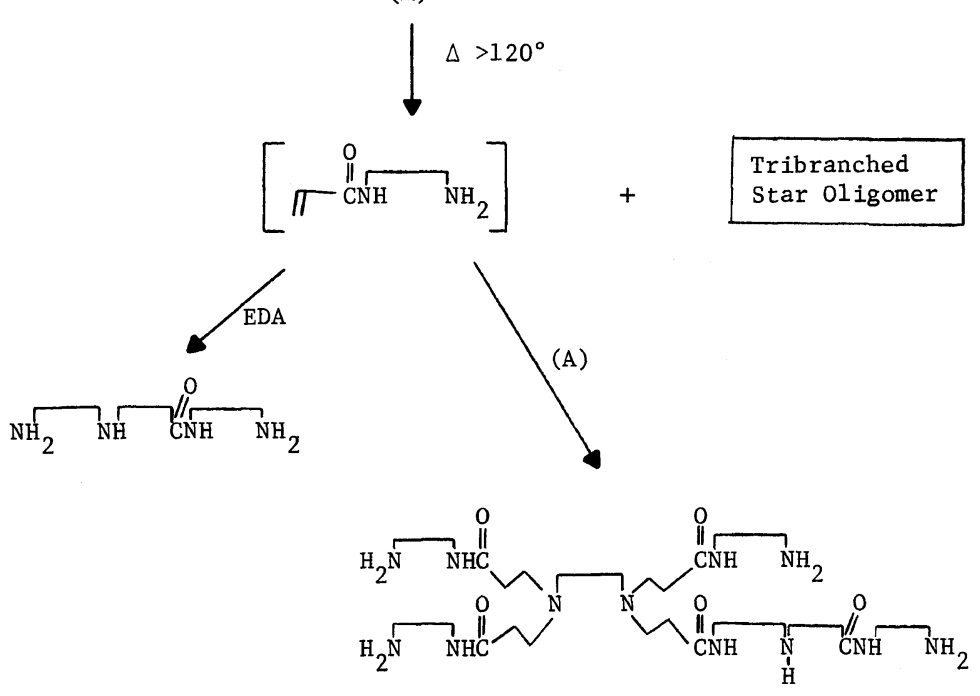

Figure 3. Reversibility of first generation dendrimer growth.

of the generation sequences. This latter factor is similar to the dendrimer fragmentation dilema described above since in either case the fragment or EDA can function as extraneous initiator cores thus broadening the molecular weight distribution significantly.

Dendrimer bridging can be dramatically affected by either; (i) the amount of sequencing reagent used or (ii) exposure of dendrimers to higher temperatures (i.e., $>150^{\circ} \mathrm{C}$ ). Since the Michael addition reaction is kinetically much faster than amidation, large excesses of methyl acrylate are not as essential for the alkylation stage. The amidation stage, however, requires large excesses in order to avoid dendrimer bridging. Statistical analysis indicates that excesses required for $\sim 95 \%$ monodispersed dendrimers vary from 15-234 molar excess for generations $1-5$, respectively $\left(\right.$ core $\left.=\mathrm{NH}_{3}\right){ }^{9}$ These excesses are essential since dendrimer amidation $v$ s. bridging amidation rates are kinetically similar. Attempts to reduce the EDA excesses invariable led to substantial amounts of dendrimer bridging as determined by size exclusion chromatography (SEC). For example, amidation of a 0.5 generation (core $\left.=\mathrm{NH}_{3}\right)$ with a $(2: 1)$ amine : ester ratio produced an insoluble gel, whereas a 15:1 excess gave a solvent soluble, homogeneous, high purity dendrimer $($ gen.$=1.0)$ as determined by SEC.

Precautions must be taken to avoid exposure of dendrimers to higher temperatures (i.e., $>150^{\circ} \mathrm{C}$ ). Molecular weight building due to dendrimer bridging was observed by SEC. Presumably the full generation dendrimer bridged via intermolecular transamidation reactions, whereas half generation dendrimers bridged by first undergoing retroMichael reactions to produce terminal amine functions which then participated in transamidation events.

Incomplete removal of ethylenediamine at any point in the dendrimer growth will lead to polydispersity since residual EDA will function as an initiator core to produce the 0.5 generation dendrimer and subsequent lower generation entities. Ethylenediamine was effectively removed from lower generation dendrimers by simple vacuum distillation at low temperature. Amine removal from higher generation dendrimers (i.e., $>$ gen. $=$ 4.0) required special techniques such as azeotropic 
( $n$-butanol) and wiped film distillation. These techniques allowed removal of amines to undetectable levels through generation $=6.0$. Entrapment of the $n$-butanol azeotroping agent occurred at generation $=7$, possibly for reasons predicted by Maciejewski. ${ }^{3}$ These details will be described elsewhere. ${ }^{7}$

\section{STARBURST DENDRIMER CHARACTERISTICS}

The dendrimers were isolated as amorphous pale yellow syrups to stiff glasses as one progressed to higher generations (i.e., $>$ gen. $=4$ ). The amorphous character of these highly branched systems is very different than the linear crystalline homologues prepared under similar conditions by Asahara. ${ }^{6}$ In all cases the neat ester-terminated (half-generation) dendrimers were lower viscosity than the amineterminated products. The dendrimers studied were soluble in most organic solvents (i.e., $\mathrm{CHCl}_{3}$, $\mathrm{MeOH}, \mathrm{DMF})$ as well as water. The ester-terminated dendrimers exhibited surface active properties in water, giving foamy solutions, in contrast to the amine-terminated products. Both the half and full generation dendrimers produced highly colored complexes with $\mathrm{CuSO}_{4}$ solutions. The ester terminated dendrimers formed deep blue complexes, whereas the amine terminated species gave deep purple colored solutions.

\section{SOLUTION PROPERTIES}

Viscosity studies conducted in a variety of solvents showed that the amine-terminated full generation dendrimers (i.e., $G=1.0,2.0,3.0,4.0$, and 5.0) generally exhibit higher intrinsic viscosities than the corresponding ester-terminated half generations (i.e., $G=1.5,2.5,3.5$, and 4.5). See Table II. Ester-terminated dendrimers could not be examined in aqueous media because of their facile hydrolysis to carboxylic acid moieties. A $\log / \log$ plot of $(\eta) v s$. molecular weight (Figure 4) illustrates this point and gives Mark-Houwink constants as shown in Table III. No doubt the higher viscosities noted for the amine $v s$. ester-terminated dendrimers are due to intermolecular hydrogen bonding (dendrimer aggregation). This is supported by the fact that $[\eta]$ determined for the generations ( $G=1.0,2.0$, and 4.0 ) in concentrated $\mathrm{HCl}$ are substantially reduced and
Table II. Intrinsic $[\eta]$ for dendrimers (core $=\mathrm{NH}_{3}$ ) in various solvents at $\left(25^{\circ} \mathrm{C}\right)$

\begin{tabular}{ccc}
\hline & & {$[\eta]$} \\
\cline { 3 - 3 } Generation & Solvent & $\mathrm{dlg}^{-1}$ \\
\hline 1.0 & $\mathrm{MeOH}$ & 0.032 \\
2.0 & $\mathrm{MeOH}$ & 0.040 \\
3.0 & $\mathrm{MeOH}$ & 0.047 \\
4.0 & $\mathrm{MeOH}$ & 0.058 \\
5.0 & $\mathrm{MeOH}$ & 0.063 \\
0.5 & $\mathrm{MeOH}$ & 0.0057 \\
1.5 & $\mathrm{MeOH}$ & 0.021 \\
2.5 & $\mathrm{MeOH}$ & 0.025 \\
3.5 & $\mathrm{MeOH}$ & 0.030 \\
4.5 & $\mathrm{MeOH}$ & 0.032 \\
1.0 & $\mathrm{H}_{2} \mathrm{O}$ & 0.031 \\
2.0 & $\mathrm{H}_{2} \mathrm{O}$ & 0.041 \\
4.0 & $\mathrm{H}_{2} \mathrm{O}$ & 0.059 \\
1.0 & $\mathrm{HCl}$ & 0.023 \\
2.0 & $\mathrm{HCl}$ & 0.023 \\
4.0 & $\mathrm{HCl}$ & 0.037 \\
\hline
\end{tabular}

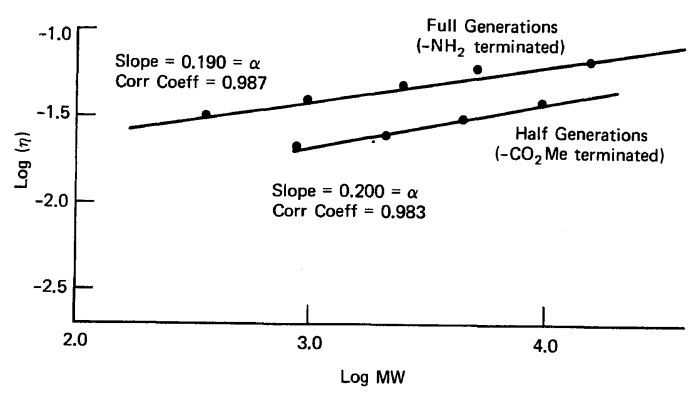

Figure 4. $\log$ intrinsic viscosity vs. $\log$ molecular weight.

Table III. Mark-Houwink constants ( $K$ and $\alpha$ ) for dendrimers $\left(\right.$ core $\left.=\mathrm{NH}_{3}\right)$ determined in various solvents

\begin{tabular}{lllc}
\hline \multicolumn{1}{c}{ Generations } & \multicolumn{1}{c}{ Solvent } & \multicolumn{1}{c}{$K$} & $\alpha$ \\
\hline $1.0,2.0,3.0,4.0,5.0$ & $\mathrm{MeOH}$ & $1.07 \times 10^{-2}$ & 0.190 \\
$1.5,2.5,3.5,4.5$ & $\mathrm{MeOH}$ & $5.25 \times 10^{-3}$ & 0.200 \\
$1.0,2.0,3.0,4.0,5.0$ & $\mathrm{Concd} \mathrm{HCl}$ & $6.3 \times 10^{-3}$ & 0.203 \\
$1.0,2.0,3.0,4.0,5.0$ & $\mathrm{H}_{2} \mathrm{O}$ & $7.76 \times 10^{-3}$ & 0.237 \\
\hline
\end{tabular}

begin to compare closely to those obtained for half generations $(G=1.5,2.5,3.5$, and 4.5$)$ in methanol. The Mark-Houwink shape factors $(\alpha=0.19 \rightarrow 0.24)$ 


\section{A. Tomalia et al.}

Table IV. Calculated hydrodynamic volumes and radii for various dendrimers (core $=\mathrm{NH}_{3}$ )

\begin{tabular}{|c|c|c|c|c|}
\hline Generation & Solvent & $\frac{[\eta]}{\mathrm{dl} \mathrm{g}^{-1}}$ & $\begin{array}{l}\begin{array}{l}\text { Hydrodynamic } \\
\text { volume, } v\end{array} \\
\begin{array}{l}\mathrm{ml} \mathrm{mol}^{-1} \\
\left(\times 10^{-21}\right)\end{array}\end{array}$ & $\begin{array}{l}\text { Hydrodynamic } \\
\text { radii } \\
\frac{r}{\AA}\end{array}$ \\
\hline 1.0 & $\mathrm{H}_{2} \mathrm{O}$ & 0.031 & 0.749 & 5.7 \\
\hline 1.0 & $\mathrm{HCl}$ & 0.023 & 0.537 & 5.1 \\
\hline 1.0 & $\mathrm{MeOH}$ & 0.032 & 0.761 & 5.7 \\
\hline 1.5 & $\mathrm{MeOH}$ & 0.021 & 1.22 & 6.6 \\
\hline 2.0 & $\mathrm{H}_{2} \mathrm{O}$ & 0.041 & 2.65 & 8.6 \\
\hline 2.0 & $\mathrm{MeOH}$ & 0.040 & 2.55 & 8.5 \\
\hline 2.5 & $\mathrm{MeOH}$ & 0.025 & 3.45 & 9.8 \\
\hline 3.0 & $\mathrm{MeOH}$ & 0.047 & 7.66 & 12.2 \\
\hline 3.5 & $\mathrm{MeOH}$ & 0.030 & 8.92 & 12.9 \\
\hline 4.0 & $\mathrm{H}_{2} \mathrm{O}$ & 0.059 & 19.1 & 16.7 \\
\hline 4.0 & $\mathrm{HCl}$ & 0.037 & 12.2 & 14.3 \\
\hline 4.0 & $\mathrm{MeOH}$ & 0.058 & 19.1 & 16.6 \\
\hline 4.5 & $\mathrm{MeOH}$ & 0.032 & 19.7 & 16.8 \\
\hline 5.0 & $\mathrm{MeOH}$ & 0.063 & 63.1 & 24.6 \\
\hline
\end{tabular}

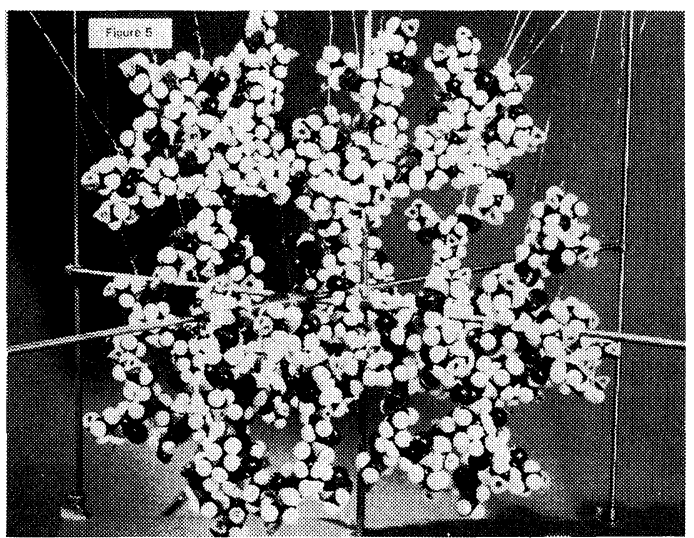

Figure 5. Corey-Pauling model for a typical dendrimer.

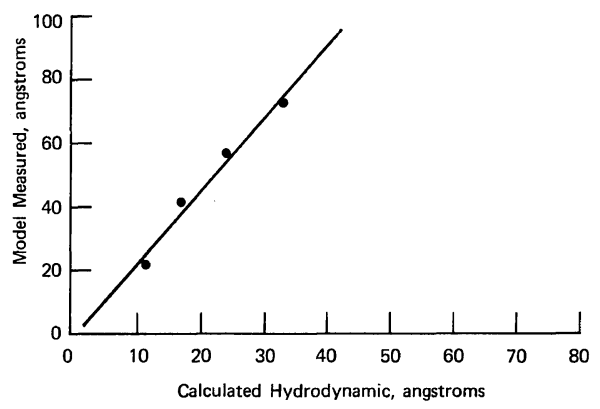

Figure 6. Comparison of diameters. compare quite favorable with the expected soft spheroidal structures these dendrimers are presumed to posses.

Assuming spheroidal architecture in solution, we calculated hydrodynamic volumes and diameters according to the Hester-Mitchell relationship shown below. These values are shown in Table IV and are compared as a function

$$
d=\left[\frac{240}{\pi N}\right]^{1 / 3}[M(\eta)]^{1 / 3}
$$

of generation with diameter values measured from Corey-Pauling (CPK) models (see Figure 5). The experimentally determined values are substantially smaller than the fully extended CPK model values, however, they are linearly related as shown in Figure 6. These contractions are presumably due to intramolecular hydrogen bonding to form more compact pseudo-cyclic type structures.

\section{ANALYTICAL METHODS USED FOR STRUCTURE VALIDATION}

The structural subtleties represented in these unique topological macromers required a variety of analytical methods for unequivocal verification. No single method could be used as conclusive proof, but rather accumulative corroboration of- 
Table V. Comparison of equivalent weights and $1^{\circ}: 3^{\circ}$ amine ratios for various dendrimers $\left(\right.$ core $\left.=\mathrm{NH}_{3}\right)$

\begin{tabular}{cccccc}
\hline \multirow{2}{*}{ Generation } & \multicolumn{2}{c}{ Equivalent weight } & & \multicolumn{2}{c}{$1^{\circ}: 3^{\circ}$ Amine ratios } \\
\cline { 2 - 3 } \cline { 5 - 5 } & Calculated & Theory & & Calculated & Theory \\
\hline 1.0 & 88.3 & 89.8 & 2.92 & 3.00 \\
2.0 & 101.3 & 104.3 & 1.56 & 1.50 \\
3.0 & 111.1 & 111.4 & 1.36 & 1.20 \\
\hline
\end{tabular}

fered by the many methods described below, was used as proof for the starburst structures. The critical dendrimer features demonstrated by these methods are outlined below, with subsequent detail in some cases.

I. Elemental Composition: $\mathrm{C}, \mathrm{H}, \mathrm{N}$ analyses, mass spectroscopy fragmentation patterns.

II. Predictable Molecular Weights vs. Generation: Low angle laser light scattering, chemical ionization and fast atom bombardment mass spectroscopy and vapor phase osmometry.

III. Homogenity: Size exclusion chromatography and electron microscopy.

IV. Interior and End Group: Infrared, ${ }^{15} \mathrm{~N},{ }^{13} \mathrm{C}$, and ${ }^{1} \mathrm{H}$ NMR spectroscopy, titrimetry and stoichometry with various reagents.

V. Topological Features: Rheology studies, electron microscopy, ${ }^{13} \mathrm{C}$ and ${ }^{1} \mathrm{H}$ NMR (relaxation times, integral ratios $v s$. generation).

VI. Dimensions: Electromicroscopy and rheology studied.

In all cases small molecule model systems were used extensively for process optimization and verification of stoichiometries. Details for the major analytical methods utilized are as follows:

\section{TITRIMETRY}

Dendrimer analysis by titrimetry was both supportive and surprising. Titration of full generations $(G=1.0,2.0$, and 3.0$)$ with $0.1 \mathrm{~N} \mathrm{HCl}$ gave sharp breaks at $\mathrm{pH} \cong 3.86$ and $\mathrm{pH} \cong 6.85$ as shown in Figure 7. The equivalents of acid required were in close agreement with theoretical values calculated for terminal primary amines and interior tertiary amines, respectively (see Table V). The surprising order of basicity (i.e., $1^{\circ}$ amine $>3^{\circ}$ amines) in these prototypes was confirmed by observing chemical shifts $\left({ }^{13} \mathrm{C}\right.$ of carbons attached to $1^{\circ}$ and $3^{\circ}$ amines,

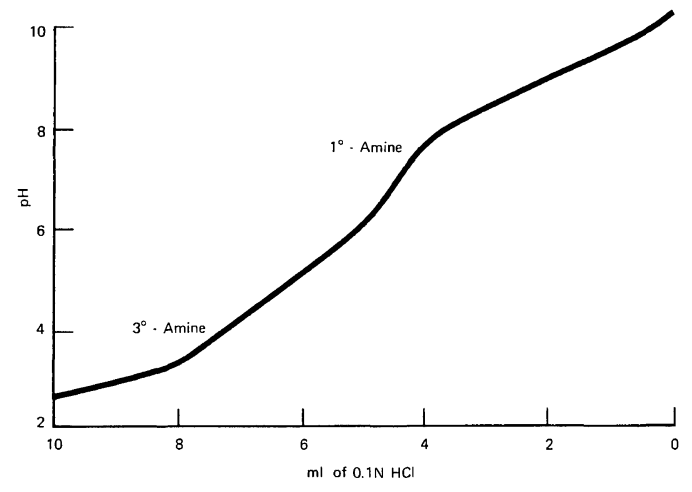

Figure 7. Dendrimer titration $\mathrm{pH} v s . \mathrm{ml}$ of $0.1 \mathrm{~N} \mathrm{HCl}$.

respectively, as a functional of $\mathrm{pH}$ ).

The distinctive endpoints exhibited by these polyamines is quite different than those observed for other amine macromolecules such as polyethyleneimine. ${ }^{10,11}$ It should be noted that recent work by Barcucci et al., ${ }^{12}$ has shown that polyamidoamines do exhibit unique titration characteristics similar to our results.

\section{MASS SPECTROSCOPY}

Chemical ionization mass spectroscopy was used extensively both for the determination of dendrimer molecular weights as well as for identification of dendrimer defect fragments during synthesis. This analytical method was satisfactory for obtaining exact parent ion peaks $(M+1)$ up to approximately 1000. Molecular ion peaks were obtained for generations $0.5-1.5$ (mol wt. $275-1000$ ) by this method. Higher molecular weight dendrimers (i.e., gen. $=2.0-4.0$; mol wt $1,000-3,000$ ) were characterized by fast atom bombardment (FAB) techniques. In all cases molecular ion peaks were in agreement with molecular weights determined by 
low angle laser light scattering as well as titrimetric methods.

\section{NUCLEAR MAGNETIC RESONANCE SPECTROSCOPY}

The dendrimers were examined by ${ }^{1} \mathrm{H},{ }^{13} \mathrm{C}$, and ${ }^{15} \mathrm{~N}$ magnetic resonance spectroscopy. Use of model compounds, integration values and analysis of relaxation times allowed complete spectral assignments. These parameters provided support for the proposed dendrimer structures as well as means for appraisal of intramolecular cyclization and intermolecular bridging processes.

Proton magnetic resonance spectroscopy for the half generations (i.e., 1.5, 2.5, 3.5, 4.5; core $=$ ethylenediamine) gave characteristic patterns with assignments as shown in Figure 8. These patterns proved to be very diagnostic not only for the structure proof but also allowed one to follow the extent of amine alkylation by methyl acrylate. For example a singlet $\left(3.60 \mathrm{ppm},-\mathrm{CO}_{2}-\mathrm{CH}_{3}\right)$ was compared to a multiplet at $(3.0-3.40 \mathrm{ppm},-\mathrm{CONH}-$ $\underline{\mathrm{CH}}_{2}{ }^{-}$) and multiplets at $\sim 2.00-3.00 \mathrm{ppm}$, (all other $\left.-\mathrm{CH}_{2}-\right)$ to determine degree of completion for the Michael addition reaction. Comparison of a broad triplet at $6.67-7.10 \mathrm{ppm}\left(\mathrm{CDCl}_{3}\right.$ or DMSO$d_{6}$ ); all noncyclic - $\mathrm{CONH}-$ ) to the various upfield resonances, served to corroborate the proposed dendrimer structures for each generation by their adherence to theoretical ratios as shown in The horizontal columns (Table VI). Development of idealized starburst branching as a function of generation was ascertained by determining equivalent proton ratios in equimolar samples of various generations. These ratios were found to be in close agreement with theoretical values shown in the vertical columns in Table VI.

Occasionally when this amide resonance was too high, it could be attributed to dendrimer bridging (structure C, Figure 8) which arose from intermolecular amidation of intermediates such as structure (A). This was especially prevalent if Michael addition reactions were conducted at higher temperatures (i.e., $>100^{\circ} \mathrm{C}$ ) or less than an excess of methyl acrylate, as determined by size exclusion chromatography. Allowing the Michael addition reaction to proceed with less than an excess of

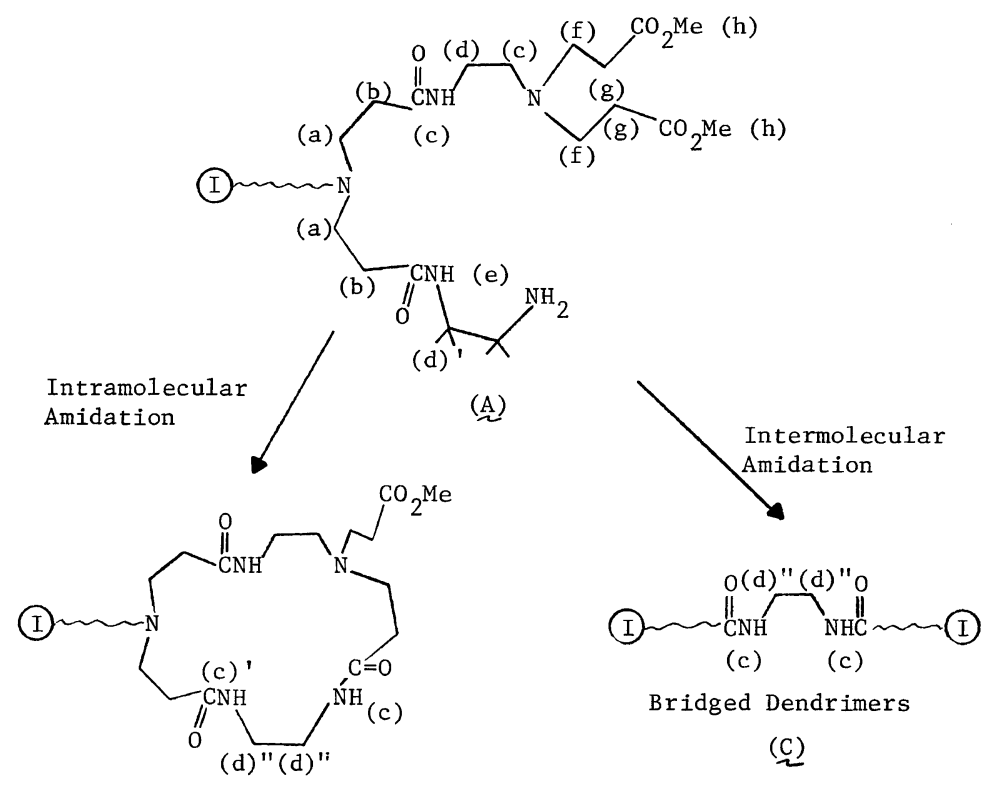

(B)

Figure 8. Intramolecular and intermolecular amidations with ${ }^{1} \mathrm{H}$ NMR assignments. (a), (b), (e), (f), (g), $\sim 2.00-3.00 \mathrm{ppm}$ (complex multiplet); (d), (d)' $(\mathrm{d})^{\prime \prime}, 3.00-3.40 \mathrm{ppm}$ (multiplet); (h), $3.60 \mathrm{ppm}$ (singlet); (c), 6.67-7.10 ppm; (c)', 7.35-7.83 ppm. 
Table VI. Half generation proton types as a function of generation (core $=\mathrm{EDA})$

\begin{tabular}{|c|c|c|c|c|c|}
\hline \multirow{2}{*}{\multicolumn{2}{|c|}{ Generation }} & \multicolumn{4}{|c|}{ Assigned proton types } \\
\hline & & (a) & (b) & (c) & (d) \\
\hline \multirow[t]{3}{*}{1.5} & a & 60 & 8 & 24 & 4 \\
\hline & $\mathrm{b}$ & $(15.0)$ & $(2.00)$ & $(6.00)$ & $(1.00)$ \\
\hline & c & {$[15]$} & [2] & [6] & {$[1]$} \\
\hline \multirow{3}{*}{\multicolumn{2}{|c|}{2.5}} & 140 & 24 & 48 & 12 \\
\hline & & $(11.67)$ & $(2.00)$ & $(4.00)$ & $(1.00)$ \\
\hline & & {$[35]$} & [6] & {$[12]$} & [3] \\
\hline \multirow{3}{*}{\multicolumn{2}{|c|}{3.5}} & 300 & 56 & 96 & 28 \\
\hline & & $(10.71)$ & $(2.00)$ & $(3.43)$ & $(1.00)$ \\
\hline & & {$[75]$} & {$[14]$} & {$[24]$} & [7] \\
\hline \multirow{3}{*}{\multicolumn{2}{|c|}{4.5}} & 620 & 120 & 192 & 60 \\
\hline & & $(10.33)$ & $(2.00)$ & $(3.20)$ & $(1.00)$ \\
\hline & & {$[155]$} & {$[30]$} & {$[48]$} & {$[15]$} \\
\hline \multirow[t]{3}{*}{5.5} & & 1260 & 248 & 384 & 124 \\
\hline & & $(10.16)$ & $(2.00)$ & $(3.10)$ & $(1.00)$ \\
\hline & & {$[315]$} & {$[62]$} & {$[96]$} & [31] \\
\hline
\end{tabular}

a Number of protons of each assigned type.

b Ratio of proton types within each generation.

c Ratio of proton type to number of amide protons in generation $=1.0$.

methyl acrylate in a highly diluted state frequently gave rise to a new downfield amide-type resonance at $7.35-7.83 \mathrm{ppm}$ (broad triplet). This has been tentatively assigned to amide groups which can assume special "inside" orientations such as illustrated by (c)' vs. (c) in structure B. Similar downfield amide resonances have also been observed by us in unreported fourteen membered macrocyclic polyamidoamines, as shown below. These structures possess "inside" ((c)' type) and "outside" ((c) type) amides which will be described in more detail elsewhere. ${ }^{7 \mathrm{~b}}$

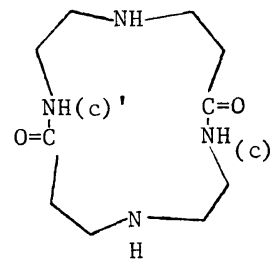

The ${ }^{1} \mathrm{H}$ NMR spectra for full generation dendrimers (i.e., 1.0, 2.0, 3.0, 4.0, 5.0; core $=$ ethylenediamine) were consistent and predictable for the proposed dendrimer growth. Table VII shows the resonance assignments which were used
Table VII. Full generation proton types as a function of generation (core $=\mathrm{EDA})$

\begin{tabular}{|c|c|c|c|c|c|c|}
\hline \multirow{2}{*}{\multicolumn{2}{|c|}{ Generation }} & \multicolumn{5}{|c|}{ Assigned proton types } \\
\hline & & (a) & (b) & (c) & (d) & (e) \\
\hline \multirow[t]{3}{*}{1.0} & a & 8 & 8 & 20 & 8 & 4 \\
\hline & $\mathrm{b}$ & $(2.00)$ & $(2.00)$ & $(5.00)$ & $(2.00)$ & $(1.00)$ \\
\hline & c & [2] & [2] & [5] & [2] & [1] \\
\hline \multirow{3}{*}{\multicolumn{2}{|c|}{2.0}} & 16 & 24 & 56 & 24 & 12 \\
\hline & & (1.33) & $(2.00)$ & $(4.67)$ & $(2.00)$ & $(1.00)$ \\
\hline & & {$[4]$} & [6] & {$[14]$} & [6] & [3] \\
\hline \multirow{3}{*}{\multicolumn{2}{|c|}{3.0}} & 32 & 56 & 116 & 56 & 28 \\
\hline & & (1.14) & $(2.00)$ & $(4.14)$ & $(2.00)$ & $(1.00)$ \\
\hline & & [8] & {$[14]$} & [29] & {$[14]$} & [7] \\
\hline \multirow{3}{*}{\multicolumn{2}{|c|}{4.0}} & 64 & 120 & 244 & 120 & 60 \\
\hline & & (1.07) & $(2.00)$ & $(4.07)$ & $(2.00)$ & $(1.00)$ \\
\hline & & {$[16]$} & {$[30]$} & {$[61]$} & {$[30]$} & {$[15]$} \\
\hline \multirow{3}{*}{\multicolumn{2}{|c|}{5.0}} & 128 & 248 & 500 & 248 & 124 \\
\hline & & $(1.03)$ & $(2.00)$ & $(4.03)$ & $(2.00)$ & $(1.00)$ \\
\hline & & [32] & [162] & [125] & {$[62]$} & [31] \\
\hline
\end{tabular}

a Number of protons of each assigned type.

b Ratio of proton types in each generation.

c Ratio of proton type to number of amide protons in generation $=1.0$.

to compare integral values as a function of generation. Both the assignments and integral values were consistent with the idealized starburst branching since the ratio of assigned protons within each generation were found to vary in a very predictable fashion according to the theoretical values (horizontal columns) shown in Table VII. Idealized dendrimer growth was ascertained by a comparison of experimentally determined proton ratios with theoretical ratios (vertical columns). Close agreement supported the proposed starburst branching.

Carbon-13 spectral data with assignments for starburst dendrimers (core $=$ ethylenediamine; generations $=0.5-2.5$ ) are shown in Table VIII. These assignments were made based on model compounds, integrated areas, relaxation measurements and corroborative ${ }^{15} \mathrm{~N}$ NMR spectral measurements. It is interesting to note that the chemical shift of given carbons in the interior generations of the dendrimers are insensitive to substitutions on the outer, terminal portions of the dendrimer. For example, the chemical shifts of carbons "a," "b," "c," "d," and "e" of generations 1.5 and 2.5 are nearly identical. Also, the shifts of the carbons in the outermost lamina, both for half 
Table VIII. Carbon-13 chemical shift assignments starburst dendrimers (core $=$ ethylenediamine: gen $=0.5-2.5$ )

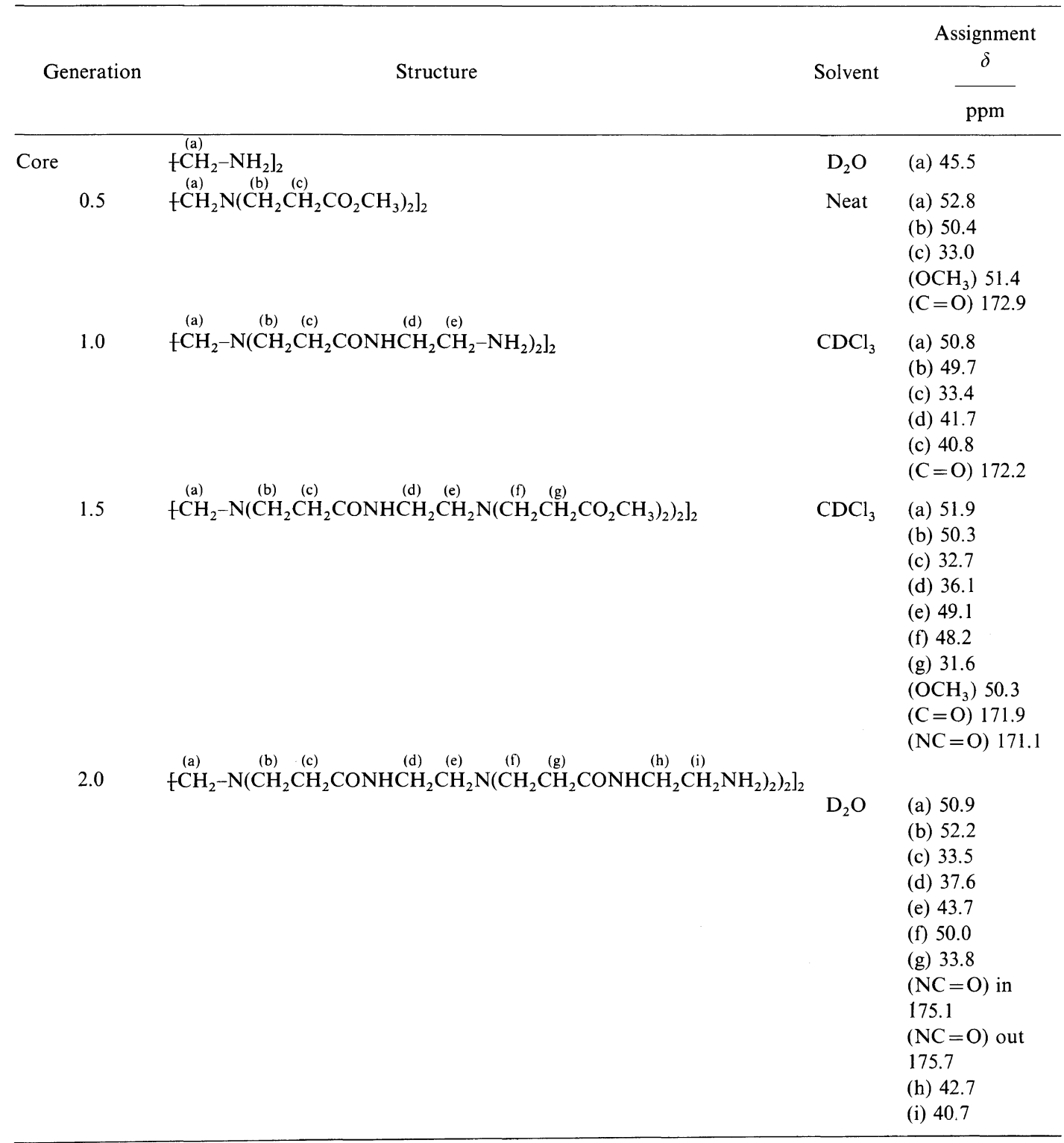

and full generations have the same chemical shifts. Compare, for example, carbons "f," "g" and the ester carbonyl of gen. $=1.5$ to carbons " $\mathrm{j}$," " $\mathrm{k}$ " and the ester carbonyl of gen. $=2.5$. Thus, the assignments and this behavior are self-consistent and support the starburst structural concept since proposed dendrimer growth would be expected to develop anisotropic homogenity around the interior carbons by virtue of their concentric symmetry.

Furthermore, relaxation times $\left(T_{1}\right)$ as measured by the inversion recovery method indicate that the interior carbons of the dendrimer are considerably less mobile than the exterior carbons. For example carbons "a," "c," and "i" (generation =2.5) have relaxation times of $<0.12,0.12$, and 0.19 seconds, respectively. Generally, relaxation times $\left(T_{1}\right)$ are 
Table VIII. (continued)

\begin{tabular}{|c|c|c|c|}
\hline Generation & Structure & Solvent & $\begin{array}{c}\text { Assignment } \\
\delta \\
\end{array}$ \\
\hline & & & $\mathrm{ppm}$ \\
\hline 2.5 & 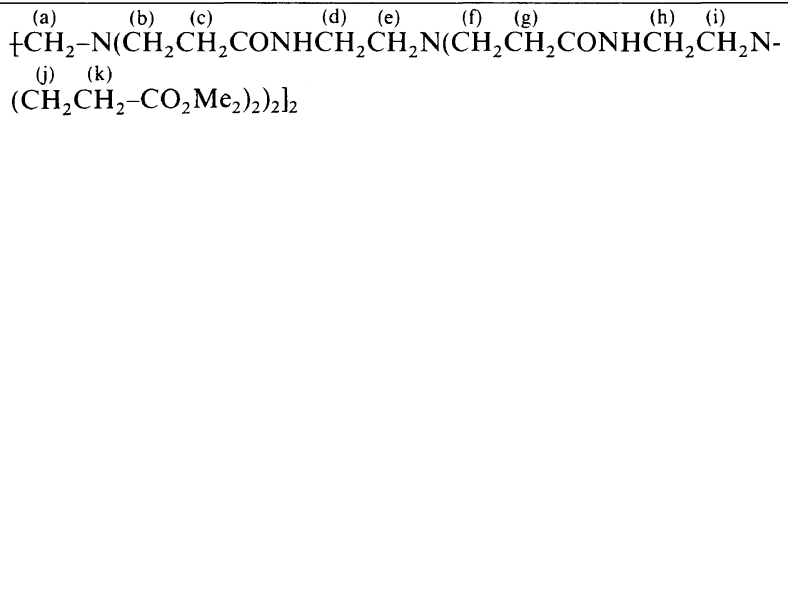 & $\begin{array}{l}\mathrm{CDCl}_{3} \\
\mathrm{CDCl}_{3}\end{array}$ & $\begin{array}{l}\text { (a) } 51.6 \\
\text { (b) } 51.9 \\
\text { (c) } 33.0 \\
\text { (d) } 36.6 \\
\text { (e) } 49.1 \\
\text { (f) } 51.9 \\
\text { (g) } 33.0 \\
\text { (h) } 36.2 \\
\text { (i) } 48.9 \\
\text { (j) } 48.4 \\
\text { (k) } 31.6 \\
\text { (OCH } 50.5 \\
\text { (NC=O) in } \\
\text { 171.3 } \\
\text { (NC=O) out } \\
\text { 171.2 } \\
\text { (C=O) } 171.8\end{array}$ \\
\hline
\end{tabular}

inversely proportional to the carbons' immobility, mobility and innermost carbons' $\left(T_{1}\right)$ would be expected to be shorter than exterior carbons. In support of the proposed dendrimer structure, the core carbon "a" has the shortest $\left(T_{1}\right)$, while the $\left(T_{1}\right)$ values become increasingly long as one proceeds to the more exterior, less immobilized lamina.

\section{LIGHT SCATTERING}

The weight-average molecular weights of various dendrimers were determined by low angle laser light scattering techniques. Solutions of full generation dendrimers $\left(\right.$ core $=\mathrm{NH}_{3}$, gen. $=1.0,2.0,3.0$, and 4.0) were at $\mathrm{pH}=11.5$ in a buffered medium, giving measured molecular weights which compared very favorabley with theoretical values (see Table IX).

\section{ELECTRON MICROSCOPY}

Initial efforts focused on methods used by Richardson $^{13}$ and Quayle ${ }^{14}$ which involved spraying techniques followed by metal shadowning. Full generation dendrimers (i.e., gen $=3.0 ;$ core $=\mathrm{NH}_{3}$ ) were examined with some success, showing substantial amounts of aggregation, accompanied by some spheroidal entities, which appear to be close in
Table IX. Measured vs. theoretical molecular weights as determined by low angle laser light scattering (LALLS) $\left(\right.$ core $\left.=\mathrm{NH}_{3}\right)$

\begin{tabular}{ccc}
\hline \multirow{2}{*}{ Generation } & \multicolumn{2}{c}{ Molecular weight } \\
\cline { 2 - 3 } & Measured & Theoretical \\
\hline 1.0 & $535^{\mathrm{a}}(359)$ & 359 \\
2.0 & 1,000 & 1,043 \\
3.0 & 2,500 & 2,411 \\
4.0 & 4,980 & 5,147 \\
\hline
\end{tabular}

a This molecular weight is too low for good experimental accuracy by the LALLS method. The molecular weight was also determined via mass spectroscopy and was identical to the theoretical.

size to extended dendrimer dimensions predicted from CPK models (i.e., $57 \AA$ vs. $39 \AA$ ). Metal shadowing techniques have been reported to give some dimensional uncertainties due to distortions for samples smaller than $100 \AA$, thus a more direct method was sought. In general the dendrimers examined were of such low molecular weight and bore such light atoms that they were virtually transparent. It was discovered, however, that coordination of terminal functionality with Group I metals (e.g., $\mathrm{Na}, \mathrm{K}, \mathrm{Cs}$ or $\mathrm{Rb}$ ) provided very 


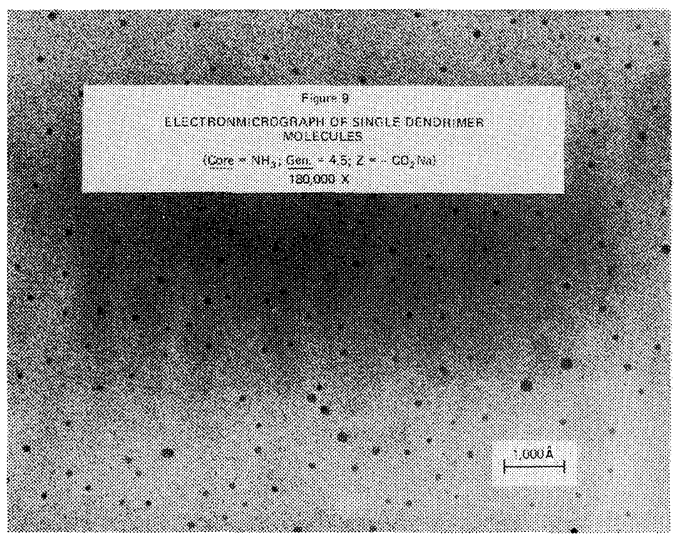

Figure 9. Electronmicrograph of single dendrimer molecules. core, $\mathrm{NH}_{3}$; gen., 4.5; $Z,-\mathrm{CO}_{2} \mathrm{Na}$. $\times 180,000$.

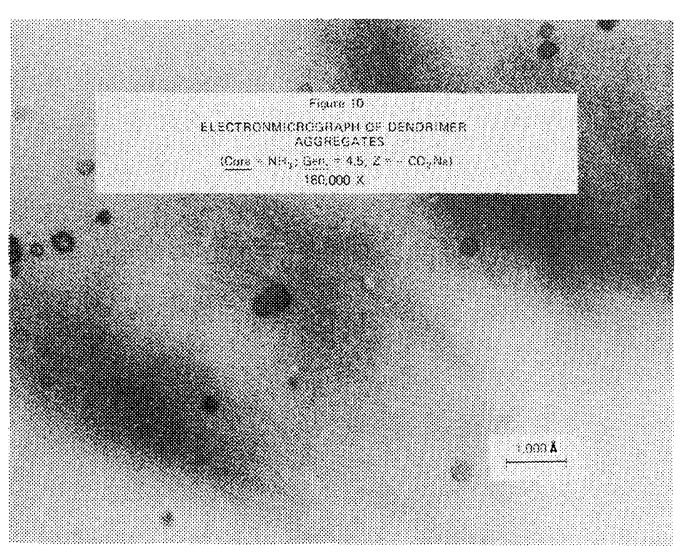

Figure 10. Electronmicrograph of dendrimer aggregates. core, $\mathrm{NH}_{3}$; gen., $4.5 ; \mathrm{Z},-\mathrm{CO}_{2} \mathrm{Na}$. $\times 180,000$.

adequate contrasting and allowed direct observation and measurement of single dendrimer molecules. Disposition of Group I metals on the dendrimer surfaces was accomplished by hydrolyzing the appropriate half generation (ester terminated) dendrimers with stoichiometric amounts of the Group I metal hydroxides. A dilute aqueous solution (i.e., generation $=4.5$; core $=\mathrm{NH}_{3}$ ) of a sodium carboxylated dendrimer was placed on a beryllium grif ( $\sim 1.5 \mathrm{~mm}$ dia. puddle) and allowed to evaporate. A concentration continuum developed from the perimeter of the puddle where single spheriodal dendrimer molecules were observed (see Figure 9), to a second region where a variety of

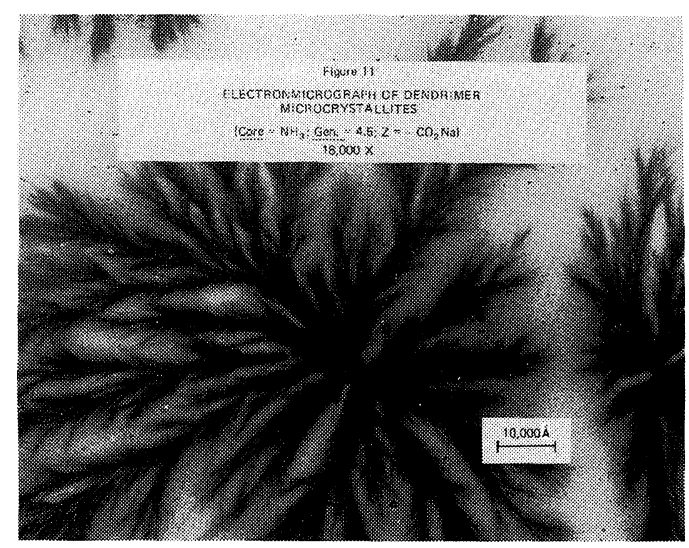

Figure 11. Electronmicrograph of dendrimer microcrystallites. core, $\mathrm{NH}_{3}$; gen., $4.5 ; Z,-\mathrm{CO}_{2} \mathrm{Na}$. $\times 18,000$.

aggregation types were noted (see Figure 10) and finally to the center where a very dendritic-like microcrystalline growth occurred in the area of highest concentration (see Figure 11).

Clustering light atoms such as sodium produced surprisingly good contrast as can be seen in Figure 9-11. Presumably an accumulative scattering effect results from the sodium atom clusters to give the contrast shown.

A particle size count of the spehroids (Figure 9) showed they were highly monodispersed with $86.6 \%$ of the particles measuring $88 \AA \pm 10 \AA$ out of a total of 47 particles. According to measurements taken from a collapsed and extended CPK model, the predicted diameter for the above dendrimer should have been $\sim 78 \AA$. Another interesting feature noted about the dendrimer in Figure 9 was that some of the spheroids were solid while others appeared to possess transparent (hollow) centers. It is believed that the first type arises as a result of flipping some of the sodium carboxylates into the center of the spheroids to produce the solid particles. Stacking the terminal functionality around the perimeter, presumably gives the annular effect observed in the second type.

The very close agreement between the observed microscopy dimensions and the predicted CPK model dimensions strongly supports the proposed dendrimer structures. Furthermore, the high selectivity and efficiency of the starburst branching process is amply demonstrated by the high degree of monodispersity observed after nine consecutive re- 
action steps (e.g., gen. $=4.5)$.

\section{CONCLUSIONS}

In conclusion, a new class of topological macromolecules referred to as "starburst polymers" has been synthesized by chemically bridging a novel type of reactive oligomer coined "dendrimers." The "time sequenced propagation techniques" used for construction of these dendrimers allows (a) controlled molecular weight building (monodispersity), (b) controlled branching (topology) and (c) versatility in design and disposition of the terminal end groups. Such molecular arrays in concert with appropriate chemical functionality have important implications in the construction of biocatalytic mimics. Finally the self replicating, symmetrical branching with multiplicity inherent in the architecture of these dendrimers provides prototypes of considerable theoretical interest for the study of fractons ${ }^{4}$ moleclar level fractals ${ }^{15 a, b}$ and topology of uniform network polymers. ${ }^{16}$

\section{EXPERIMENTAL}

\section{Materials}

All materials were redistilled and dried over molecular sieves. Ethylenediamine (Dow Chemical Co.) and methyl acrylate (MEHQ inhibited, Celanese Co.) were used for the synthesis. Solvents were spectroscopic grade, distilled in glass (Burdick and Jackson Labs) and used as is.

\section{Synthesis}

Both the Michael addition reaction and the amidation reactions were conducted in methanolic solvent at temperatures below $50^{\circ} \mathrm{C}$. A slight methyl acrylate $(\cong 10 \mathrm{~mol} \%)$ excss was used for the alkylation step, however, very large excesses of ethylenediamine (i.e., 15-250 (EDA : ester) molar ratios) were necessary to prevent dendrimer bridging and gelation. Excess reagents and solvent were removed under high vacuum $(\cong 0.075 \mathrm{mmHg})$ at temperatures below $55^{\circ} \mathrm{C}$. Progress of the reaction was monitored by infrared, NMR and mass spectroscopy as well as by size exclusion chromatography. Half generation dendrimers were monitored by SEC as THF solutions whereas full generations were examined as aqueous solutions. Gas chromatography was used routinely to determine the pre- sence of "sequence propagating reagent," (i.e., EDA or MA) in the respective dendrimer samples. More complete synthesis details will be reported elsewhere. $^{7}$

\section{Analytical Methods and Instrumentation}

Light scattering measurements were made on a Chromatrix KMX-6 $\left(0.2 \mathrm{~mm}\right.$ field stop with $6-7^{\circ}$ annulus) using $0.1 \mathrm{M} \mathrm{Na}_{2} \mathrm{HPO}_{4}$ buffer solutions of dendrimer $\left(1-25 \mathrm{mg} \mathrm{ml}^{-1}\right)$. Specific refractive index increments were determined with a Chromatrix KMX-16 differential refractometer.

Size exclusion chromatographic analyses of the full generations involved aqueous solutions of dendrimer injected as $100 \mu \mathrm{l}(0.5 \%$ solutions $)$ samples onto Spherogel TSK/PW 2000, $3000(60 \mathrm{~cm})$ columns with a $0.05 \mathrm{M} \mathrm{K}_{2} \mathrm{HPO}_{4}(\mathrm{pH}=11$ with $\mathrm{NaOH})$ eluent $\left(1 \mathrm{ml} \mathrm{min}{ }^{-1}\right)$. A Hewlett Packard 1081B differential refractive index detector was used and interfaced with a Hewlett Packard 85 computer via a Nelson Analytical Model A/D converter. The half generation dendrimers were analyzed as THF solutions $(1.0 \%)$ pumped at $1 \mathrm{ml} \mathrm{min}^{-1}$ through a $100 \AA \mu$-Styrogel column $(60 \mathrm{~cm})$ and monitored by either an LC $75 \mathrm{UV}$ or differential refractive index detector system. GC analyses of residual volatiles were performed on a Hewlett-Packard 5700A chromatograph equipped with $10^{\prime} \times 1 / 8^{\prime \prime}$ glass columns packed with $60 / 40$ mesh Texax.

Carbon-13 and ${ }^{1} \mathrm{H}$ NMR spectra were obtained on a Jeol FX-60 and Varian T-60 spectrometers with dioxane or tetramethylsilane as internal references, respectively. Infrared spectra were obtained on a Perkin-Elmer Model 598 spectrometer.

Chemical ionization mass spectroscopy work was conducted with a Finnigan Model 4021 GC mass spectrometer using a direct probe technique with $\mathrm{CH}_{4}$ as the reagent gas, temperature programmed from $30-350^{\circ} \mathrm{C}$. Fast atom bombardment (FAB) masses were obtained with a Finnigan TSQ (Triple Quad) Model 4500 system.

Electron micrographs were recorded on a Philips 400 TEM microscope using spraying and metal shadowing techniques as described earlier.

Direct observation of Group I metal coordinated dendrimers was conducted on a JEM-1200EX electronmicroscope (JEOL) using CTEM techniques on a beryllium grid coated with $50 \AA$ carbon. Using STEM and energy dispersive spectroscopy (EDS) procedures it was determined that the particles 


\section{A. Tomalia et al.}

examined contained only the Group I metal intended and in amounts appropriate for the predicted dendrimer stoichiometry.

Carbon, hydrogen, and nitrogen values were in good to excellent agreement with the theoretical values.

Acknowledgements. We wish to acknowledge the dedicated support and very valuable discussions provided by Dr. P. Kirchhoff, D. Beaman, A. Vogel, R. Willency, R. A. Bruzewski, V. Berry, and R. Yocum.

\section{REFERENCES}

1. P. G. DeGennes and H. Hervet, J. Phys. Lett., 44, 351 (1983).

2. W. Burchard, K. Kajiwara, and D. Nerger, J. Polym. Sci., Phys. Ed., 20, 157 (1982).

3. M. Maciejewski, J. Macromol. Sci., Chem., A17(4), 689 (1982).

4. S. Alexander and R. Orbach, J. Phys. Lett., 43, 625 (1982).

5. This terminology was adopted as an extension of present nomenclature which refers to present radial branched systems as "star polymers." It should be noted that some scientists refer to the present systems as "cauliflower polymers." (See ref 1.)

6. T. Asahara, Makromol. Chem., 136, 211 (1970).

7. a. D. A. Tomalia, J. R. Dewald, M. Hall, P. Smith, and S. Martin, J. Org. Chem., to be published.

b. D. A. Tomalia and L. Wilson, unpublished work.

8. S. I. Suminov and A. N. Kost, Russian Chem. Rev.,
38, 884 (1969).

9. Statistical analysis shows that the mole fraction of unbridged dendrimer species is given by:

$$
f_{0}=(1-P)^{N_{\mathrm{c}} N_{r}^{G-1}}
$$

where: $P=1 /(4 X-1)$ and $X$ is the moles of ethylenediamine per mol of ester terminal group in the dendrimer. The exponential-exponential form of the above equation shows the strong dependence of $f_{0}$ on $G$. Therefore, very high $X$ values (low $P$ ) are essential for the higher generations, to assure unbridged, highly monodispersed dendrimers. A complete description with experimental verification of these statistical predictions will appear elsewhere (S. Martin et al.)

10. D. Horn, "Polymeric Amines and Ammonium Salts," Pergamon Press, Oxford, New York, Toronto, Sydney, Paris, 1979, p 333.

11. D. A. Tomalia and G. Killat, "Alkylenimine Polymers," John Wiley \& Sons, New York, N. Y., 1984 , in press.

12. R. Barbucci, M. Casolaro, P. Ferruti, V. Barone, and F. Lelj Lolivia, Macromolecules, 14, 1203 (1983).

13. M. J. Richardson, Proc. R. Soc., London Ser. A, 279, 50 (1964).

14. D. V. Quayle, Nature, London, 209, 5025 (1966).

15. a. H. J. Stapleton, J. P. Allen, C. P. Flynn, D. G. Stinson, and S. R. Kurtz, Phys. Rev. Lett., 45, 1456 (1980).

b. B. B. Mandelbrot, "The Fractal Geometry of Nature," W. H. Freeman and Co., San Francisco, 1983.

16. L. M. Chepel, V. A. Topolkaroyev, A. N. Zelenstskii, E. V. Prut, G. M. Trofinova, D. D. Novikov, and Al. A1. Berlin, Polym. Sci. U.S.S.R., 24, 1873 (1982). 